\title{
Isolated cortical vein thrombosis complicating orbital cellulitis
}

\author{
Vivek S. Murumkar' ${ }^{1}$ Karthik Kulanthaivelu' ${ }^{1}$, Sheetal Goyal' ${ }^{2}$, \\ Shamick Biswas ${ }^{1}$ \\ 'Department of Neuroimaging and Interventional Radiology, National Institute \\ of Mental Health and Neurosciences, Bangalore, Karnataka, India; ${ }^{2}$ Department \\ of Neurology, National Institute of Mental Health and Neurosciences, Bangalore, \\ Karnataka, India
}

\begin{abstract}
Background: Orbital cellulitis is characterized by the infective inflammation of orbital structures, usually posterior to the orbital septum. Extension of infection from the paranasal sinuses is the most common etiology for orbital cellulitis. Intracranial complications of orbital cellulitis include meningitis, subdural empyema, brain abscess, and cavernous sinus thrombosis.
\end{abstract}

Case presentation: A 33-year-old man presented with acute onset of foul-smelling mucopurulent nasal discharge and swelling of the left eye followed by altered sensorium. On examination of the left eye, chemosis, eyelid edema, and proptosis were present. Computed tomography (CT) of the brain revealed non-axial left proptosis with inflammatory reticulation in the intra- and extraconal fat alongside sinusitis. Magnetic resonance imaging (MRI) of the brain confirmed the CT findings and additionally showed meningitis and subdural empyema along the left frontoparietal convexity with parenchymal signal changes, suggesting venous infarction in the left frontal lobe. Susceptibility weighted imaging (SWI) confirmed the thrombus in the frontal polar vein on the left side, suggesting the diagnosis of septic isolated septic cortical venous thrombosis (ICVT) as a complication of orbital cellulitis. Cerebrospinal fluid study showed polymorphonuclear cell pleocytosis with elevated protein and lowered sugar. Blood and conjunctival swab cultures were negative. He was subsequently treated with intravenous broad-spectrum antibiotics and antifungals to which he responded and was discharged in stable condition.

Conclusions: Our case highlights the presentation of septic ICVT complicating orbital cellulitis and paranasal sinusitis. It also underscores the higher sensitivity of SWI as a crucial tool in diagnosing ICVT. Appropriate and prompt medical treatment in orbital cellulitis can prevent further complications.

Keywords: cortical vein thrombosis, empyema, meningitis, orbital cellulitis

Correspondence: Dr. Karthik Kulanthaivelu, DM, FRCR, Assistant Professor, Department of Neuroimaging and Interventional Radiology, 3rd Floor, Faculty Block, Neurocentre, National Institute of Mental Health and Neurosciences (NIMHANS), Hosur Road, Bangalore 560029, India.

E-mail: pammalkk@gmail.com! 


\section{Introduction}

Orbital cellulitis is a frequently encountered clinical condition that is potentially vision and life-threatening. The routes for the infection of orbital contents include the contiguous extension (from infected sinuses, dacryocystitis) and hematogenous spread of the systemic infections.' It frequently affects children and young adults. Typical clinical features include acute onset proptosis, lid edema, chemosis, and painful ophthalmoplegia. Left untreated, it can cause dreaded complications such as vision loss, meningitis, subdural empyema, brain abscess, and cavernous sinus thrombosis. ${ }^{2,3}$ Imaging plays a crucial role in orbital cellulitis, adding not only certainty to the diagnosis but also providing information regarding the extent of spread. Being superior in its soft-tissue contrast, magnetic resonance imaging (MRI) is the preferred modality. ${ }^{4}$ Isolated cortical vein thrombosis (ICVT) is an infrequent disease with etiologies ranging from hypercoagulable state, infections, and intracranial hypotension. 5,6 On account of its varied clinical presentation (due to differences in the afflicted anatomical region) and lack of gold-standard investigations, ICVT is an under-reported entity and often overlooked. ${ }^{5,7}$ We herein present our observations in a case of ICVT that complicated orbital cellulitis.

\section{Case report}

A 33-year-old man presented with foul-smelling mucopurulent nasal discharge for 15 days with gradually progressive swelling of the left eyelid, painful ocular movement, excessive lacrimation and reddening of the left eye for 2 days along with altered sensorium for 1 day. He was not a known diabetic or hypertensive. Pupils were equal and reactive bilaterally. Both fundi were normal on examination. Investigations revealed severe leukocytosis $(27.3 \times 1000 / \mu \mathrm{L})$ with neutrophilia and thrombocytosis. Testing for liver function, renal function, and serum electrolytes was normal. Cerebrospinal fluid (CSF) study showed a massive cellular pleocytosis of 9,600 cells $/ \mathrm{mm}^{3}$ with $85 \%$ polymorphonuclear cells. CSF gram stain showed no evidence of bacteria. A high protein $(183 \mathrm{mg} / \mathrm{dl})$, high lactate $(64.5 \mathrm{mg} / \mathrm{dl})$, and normal glucose $(70 \mathrm{mg} / \mathrm{dl})$ were the other findings. Blood and conjunctival swab cultures were negative.

Computed tomography (CT) showed proptosis in the left eye with thickening of the preseptal soft tissue, as well as intraconal and extraconal orbital fat. Mucosal thickening with hyperattenuation of ethmoid air cells was also noted (Fig. 1a). MRI of the brain confirmed the same with a better demonstration of the inflammatory process and an evolving abscess involving the extraconal and intraconal fat as well as the extraocular muscles with ethmoid and frontal sinusitis (Fig. 1b-f). The diffusion-weighted image of the orbits showed restriction (Fig. 1c and 1d), suggesting abscess formation. The postcontrast study revealed diffuse pachymeningeal and leptomeningeal enhancement along the left frontoparietal region, 


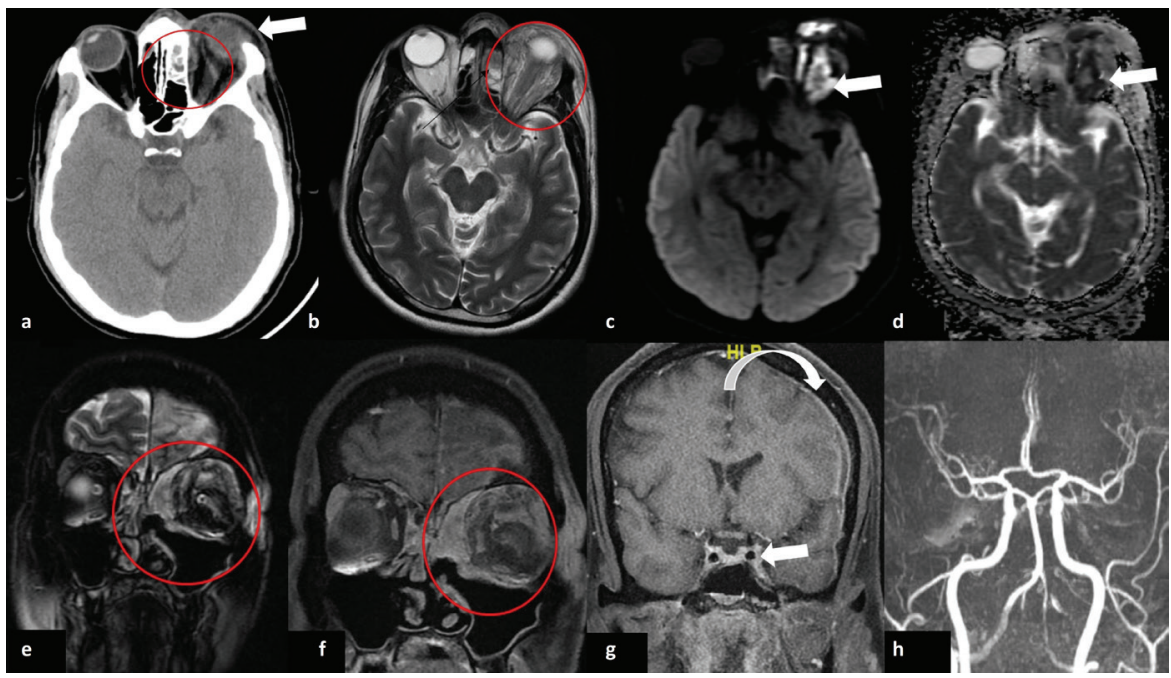

Fig. 1. (a) Axial computed tomography of the orbit showing proptosis of the left eye with thickened preseptal soft tissue (white arrow). The adjoining ethmoid air cells show hyperattenuating mucosal thickening with inflamed intraorbital fat (red circle) adjacent to the lamina papyracea. (b) Axial T2 weighted imaging (T2WI) showing inflamed intra- and extraconal fat of the left orbit with proptosis and thickened preseptal soft tissue (red circle). Note the mucosal thickening of the left ethmoid air cells with relative hypointense signal (black arrow). (c, d) Diffusion-weighted imaging and apparent diffusion coefficient (ADC) maps showing restricted diffusion (white arrows) in the infective soft tissue pointing towards abscess formation. (e,f) Coronal T2WI fat-suppressed image (e) and coronal T1WI postcontrast (T1PC) (f) showing infective soft tissue extending from the left ethmoid air cells into the left orbit (red circles). (g) T1PC at the cavernous sinus level demonstrating normal appearance of sinuses (white arrow), thereby excluding cavernous sinus thrombosis. Note the pachymeningeal enhancement (curved arrow) along the cerebral convexity. (h) Time of flight (TOF) MR angiography showing normal morphology and course of the intracranial arteries.

suggesting meningitis. The cavernous sinuses were normal (Fig. 1g). MR angiography (MRA) revealed normal course and morphology of the intracranial arteries (Fig. 1h). Cortical/juxtacortical expansile, T2/FLAIR hyperintense signal changes with foci of blooming, suggestive of hemorrhagic venous infarct involving the left anterior frontal pole, anterior cingulate gyrus, and superior frontal gyrus were seen. Subdural empyema was noted along the left frontoparietal convexity (Fig. 2a-d). Susceptibility weighted imaging (SWI) showed accentuated blooming with a serpentine morphology in the sulcal space adjoining the infarct traceable until the superior sagittal sinus, suggestive of cortical venous thrombosis (Fig. 2e and 2f). Based on the above findings, the diagnosis of left orbital cellulitis (possibly of fungal or bacterial etiology), complicated by left frontal polar cortical vein thrombosis, hemorrhagic venous infarction, and subdural empyema was made. 


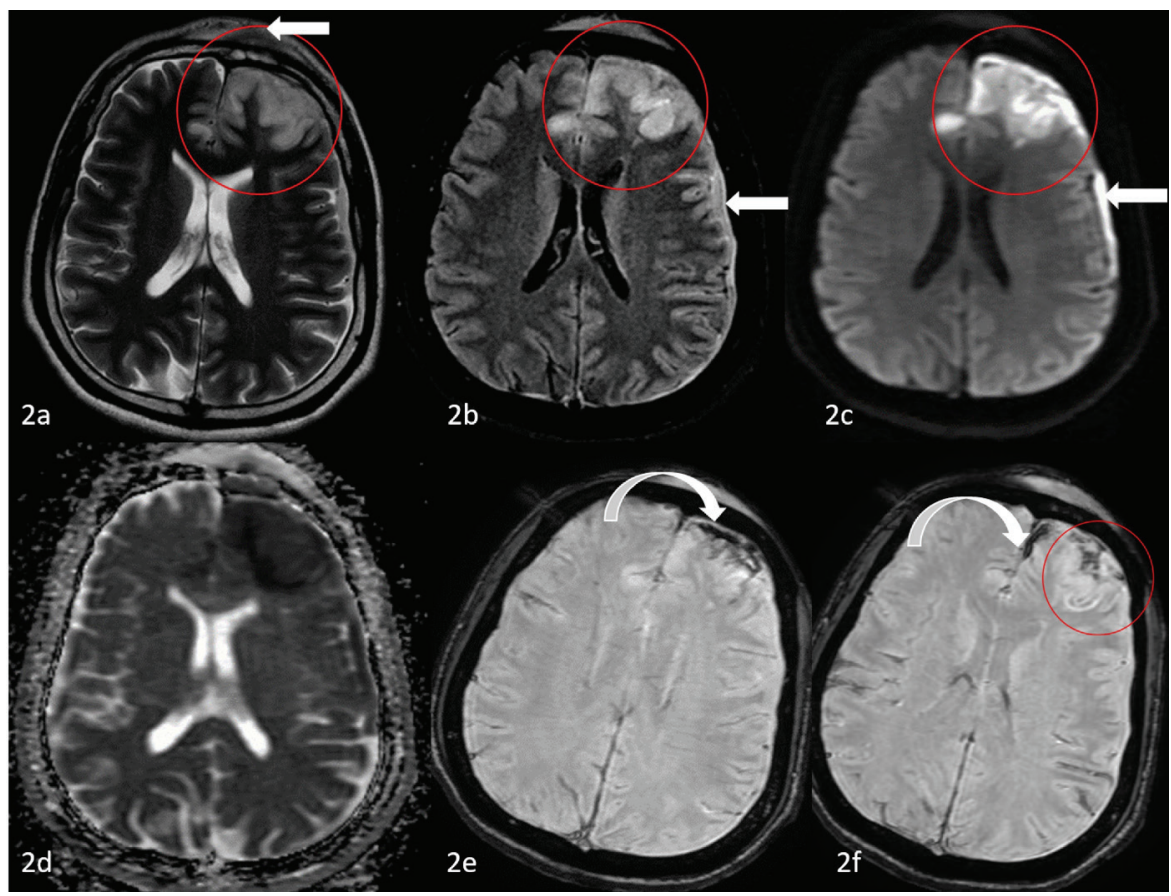

Fig. 2. (a, b) Axial T2 weighted imaging (a) and FLAIR (b) showing hyperintensity involving the left frontal lobe (red circle) with inflamed soft tissue in the subgaleal region (white arrow). Note the subdural fluid collection along the left frontoparietal region (white arrow in 2b). (c, d) Axial diffusion-weighted images and apparent diffusion coefficient images showing diffusion restriction in the involved area, suggestive of acute infarction. Diffusion restriction is also noted in the subdural fluid collection, suggestive of empyema (arrow in c). (e, f) Susceptibility weighted imaging showing serpentine hypointense (susceptibility) structure running in the sulcal space adjacent to the infarct, indicative of thrombosed vein (curved arrows). Note the multiple punctate hemorrhagic foci within the infarcted area (red circle in $\mathrm{f}$ ).

The patient was treated with a triple injectable antibiotic regimen of ceftriaxone (4 gm/day), vancomycin (1.5 gm/day), and metronidazole $(1.5 \mathrm{gm} /$ day) along with injectable hydrocortisone and antifungal amphotericin B (5 mg/kg/day). He responded to treatment and was discharged in stable condition.

\section{Discussion}

Orbital cellulitis is one of the most common ophthalmological emergencies, which unless treated carries the risk of permanent vision loss and intracranial complications. ${ }^{3}$ It commonly arises as a consequence of paranasal sinus infections. Less common etiologies include dacryocystitis, eyelid infections, and 
postsurgical complications. Rarer still are hematogenous origins and penetrating ocular trauma. ${ }^{1,2}$ It affects children and young adults predominantly. Causative agents vary with age of presentation. In children, the causative microbe is usually a single pathogen among Staphylococcus aureus, Streptococci pyogenes, and Streptococcus pneumoniae. In adults, orbital cellulitis is usually of polymicrobial origin. ${ }^{2}$ Chandler et al. classified orbital cellulitis into five different stages in increasing order of severity and morbidity. ${ }^{8}$ Group I is preseptal cellulitis, while Group II presents an inflammatory process extending into the orbit. Surgical management is warranted in Groups III and IV, which are characterized by abscess formation in the subperiosteal and intraorbital compartments. Group V is characterized by cavernous sinus thrombosis. ${ }^{1,8}$

Our index case also had a history of mucopurulent nasal discharge preceding the typical clinical features of orbital cellulitis, as has been cited earlier. Infrequently, in patients with underlying immunocompromised state such as retroviral infection and diabetes mellitus, signs of inflammation may be subtle. ${ }^{1}$ Diagnosis is often made based on history and examination findings. Imaging of the orbits, brain, and paranasal sinuses is usually required to define anatomical extensions, and more importantly, intracranial extension. Imaging also serves as a handy tool to rule out other mimics and unsuspected foreign bodies. Sinus X-rays can show air-fluid levels within the paranasal sinuses. High-frequency probe ultrasound may help in demonstrating orbital abscess. Contrast-enhanced CT is the mainstay of imaging in orbital cellulitis. CT interpretation should be done after careful analysis of both soft tissue and bone window. On account of its superior soft-tissue resolution, MRI overscores CT in terms of demonstrating the intracranial and cavernous extensions of the disease pathology, which carries worse prognosis and warrants emergency measures. ${ }^{4}$ Diffusion-weighted imaging (DWI), as has been demonstrated in our case, allows confident diagnosis of an abscess and may obviate the need to use intravenous gadolinium-based contrast media. ${ }^{4}$

Medical management with intravenous broad-spectrum antibiotic therapy is advocated. ${ }^{1,2}$ Surgical treatment is offered to patients who fail to respond to medical therapy, those with intraorbital foreign body, and those with systemic sepsis. ${ }^{1}$ Intracranial complications of orbital cellulitis include meningitis, subdural empyema, abscess, and cavernous sinus thrombosis. ${ }^{1,2,3}$ In our case, blood and conjunctival swab cultures were negative. Hyperattenuation within the ethmoid air cells coupled with the $T 2$ hypointense signal raised the possibility of a fungal etiology. ${ }^{9}$ Our patient received intravenous broad-spectrum antibiotics as well as antifungals, to which he responded with a gradual reduction in proptosis, swelling, and redness of the eyelid. Cavernous sinus thrombosis has been reported as a complication of paranasal sinusitis as well as orbital cellulitis. ${ }^{1,2,10,11}$ Direct as well as indirect venous drainage of orbital and facial structures into the 
cavernous sinus and trabeculated nature of the sinus traps emboli and microbes, predisposing its involvement. ${ }^{12}$ To the best of our knowledge, isolated septic ICVT with concomitant hemorrhagic venous infarction in the background of due orbital cellulitis has not been reported in the literature. In our case, a large hemorrhagic infarct was seen involving the left frontal polar region, anterior portions of the cingulate gyrus, and superior frontal gyrus; it was non-territorial in distribution (distinguishing it from arterial infarct). Additionally, the thrombosed frontal polar vein was seen on SWI as a serpentine cord-like structure running adjacent to the sulcal space, thus confirming the diagnosis. As with our case, SWI has been proven handy in diagnosing this rare phenomenon of ICVT. ${ }^{5}$

We wish to reflect on the origin of frontopolar vein thrombosis. Vascular complications are a relatively known phenomenon in cases of meningitis, particularly of tubercular origin, the most frequent being perforator arterial infarcts. ${ }^{13}$ Arterial damage found in meningitis can be infiltrative, proliferative, or necrotizing. Similar changes can be seen in the cortical veins. ${ }^{13}$ We thus postulate that the focal left frontal meningitis secondary to the osteodural breach that complicated the orbital cellulitis in our case could have caused septic thrombophlebitis of the frontal polar vein. Alternatively, the potential of subdural empyema as an inciting agent leading to ICVT also ought to be considered. Subdural empyema causing dural venous sinus thrombosis has been reported earlier as a complication in up to $10 \%$ of the cases. ${ }^{14}$ Diploic veins serving as the route of extension of infection from the paranasal sinusitis culminating in cortical venous thrombosis is known. Such a diploic venous route of transmission of the infection cannot be ruled out in the index case.

Treatment of septic cerebral venous thrombosis (CVT) includes maintaining optimal hydration, antimicrobial therapy targeted towards the causative agent, and surgical debridement in appropriate clinical settings. Consensus about the use of anticoagulant and corticosteroids in septic CVT is lacking. ${ }^{12,15}$

To conclude, our case highlights the presentation of septic ICVT complicating orbital cellulitis and paranasal sinusitis. It also underscores the higher sensitivity of SWI as a crucial tool in diagnosing ICVT. Appropriate and prompt medical treatment in orbital cellulitis can prevent further complications.

\section{Acknowledgments}

The authors wish to disclose they have no conflict of interest, financial interests, or have had financial support in regard to this article. 


\section{References}

1. Hegde R, Sundar G. Orbital cellulitis: A review. TNOA J Ophthalmic Sci Res. 2017;55(3):211-219.

2. Chaudhry IA, Al-Rashed W, Arat YO. The hot orbit: orbital cellulitis. Middle East Afr J Ophthalmol. 2012;19(1):34-42.

3. Chaudhry IA, Shamsi FA, Elzaridi E, Al-Rashed W, Al-Amri A, Al-Anezi F, Arat YO, Holck DE. Outcome of treated orbital cellulitis in a tertiary eye care center in the middle East. Ophthalmology. 2007;114(2):345-354.

4. Sepahdari AR, Aakalu VK, Kapur R, et al. MRI of orbital cellulitis and orbital abscess: the role of diffusion-weighted imaging. Am J Roentgenol. 2009;193(3):W244-50.

5. Singh R, Cope WP, Zhou Z, De Witt ME, Boockvar JA, Tsiouris AJ. Isolated cortical vein thrombosis: case series. J Neurosurg. 2015;123(2):427-433.

6. Stam J. Thrombosis of the cerebral veins and sinuses. N Engl J Med. 2005;352(17):1791-1798.

7. Boukobza M, Crassard I, Bousser MG, Chabriat H. MR imaging features of isolated cortical vein thrombosis: diagnosis and follow-up. Am J Neuroradiol. 2009;30(2):344-348.

8. Chandler JR, Langenbrunner DJ, Stevens ER. The pathogenesis of orbital complications in acute sinusitis. The Laryngoscope. 1970;80(9):1414-1428.

9. Aribandi M, McCoy VA, Bazan III C. Imaging features of invasive and noninvasive fungal sinusitis: a review. Radiographics. 2007;27(5):1283-1296.

10. Chang YS, Chen PL, Hung JH, et al. Orbital complications of paranasal sinusitis in Taiwan, 1988 through 2015: Acute ophthalmological manifestations, diagnosis, and management. PloS One. 2017;12(10):e0184477.

11. Schupper AJ, Jiang W, Coulter MJ, Brigger M, Nation J. Intracranial complications of pediatric sinusitis: identifying risk factors associated with prolonged clinical course. Int J Pediatr Otorhinolaryngol. 2018;112:10-15.

12. Khatri IA, Wasay M. Septic cerebral venous sinus thrombosis. J Neurol Sci. 2016;362:221-227.

13. Chatterjee D, Radotra BD, Vasishta RK, Sharma K. Vascular complications of tuberculous meningitis: An autopsy study. Neurol India. 2015;63(6): 926-932.

14. Konar S, Gohil D, Shukla D, et al, Arimappamagan A, Devi BI. Predictors of outcome of subdural empyema in children. Neurosurg Focus. 2019;47(2):E17.

15. Kojan S, Al-Jumah M. Infection related cerebral venous thrombosis. J Pak Med Assoc. 2006;56(11):494-497. 